\title{
BINARY, PULSATING, AND IRREGULAR VARIABLES AMONG PLANETARY NUCLEI
}

\author{
Howard E. Bond \\ Space Telescope Science Institute \\ Robin Ciardullo \\ Kitt Peak National Observatory
}

\section{CCD Photometry of Planetary Nuclei}

For the past two years, the authors have been carrying out a program of CCD photometry of planetary-nebula nuclei (PNNs), using the $0.9-\mathrm{m}$ telescopes at Kitt Peak National and Cerro Tololo Inter-American Observatories. The aim of this program is to investigate the variability of PNNs on timescales of minutes to days, in order to search for close binaries and pulsators, as well as unexpected new classes of variable stars.

The program represents an extension of the photoelectric photometry of PNNs carried out by Bond and Grauer (1987). Use of the two-dimensional CCD detector allows us to model and subtract the nebulosity surrounding a PNN, in addition to providing exactly simultaneous observations of the PNN and several nearby comparison stars. The latter allow us to compensate for variable atmospheric transparency, permitting accurate differential photometry to be obtained even when the observing conditions are not photometric.

\section{Close-Binary Nuclei}

One of us has recently reviewed the subject of close-binary PNNs (Bond 1988a). That review lists the seven PNNs that are photometrically confirmed close binaries with orbital periods less than one day. Two of these objects-the nuclei of Abell 46 and Abell 63-are eclipsing binaries. For the remaining five systems, the light variability is due only to heated hemispheres on the main-sequence companions of the very hot primary stars. An example of the latter type of binary is the central star of Kohoutek 1-2, which has an orbital period of 16.2 hours. A complete $B$ light curve, obtained with a CCD at CTIO, is shown below. The amplitude of the variation in the optical band is enormous, 1.2 mag peak-to-peak, because of the fact that the primary star radiates mainly in the ultraviolet, while the considerably cooler heated hemisphere radiates mainly in the optical. The central star of HFG 1 shows a nearly identical light curve, at an orbital period of 14.0 hours (Bond et al. 1988).

As discussed by Bond (1988a), the results of photometric searches for close-binary PNNs by several authors indicate that the fraction of binaries with $P \lesssim 1$ day among PNNs is about $15 \%$. If we adopt the scenario suggested by Paczynski (1976), in which a wide binary drastically reduces its orbital separation by first entering a "common-envelope" stage, and then ejecting the envelope, it becomes clear that a substantial fraction of planetary nebulae are ejected by binary interactions, rather than in the normal course of single-star evolution.

The fraction of planetary nebulae ejected from binary PNNs could be much higher if many of the nuclei are binaries with periods of days to weeks. Such binaries would have been difficult to detect in existing surveys. We are now making a special effort to monitor PNNs over multi-night intervals, and there are already suggestions that many longer-period binaries may indeed exist. Two new examples are the nucleus of $S p 1$, which we find to vary by about $0.1 \mathrm{mag}$ with a period of about 3 days (a composite spectrum had already been noted by Méndez et al. 1988), and the nucleus of Sh 2-71, which varies by $0.4 \mathrm{mag}$ with a period of approximately 2 weeks (the variability having been noted earlier by Kohoutek 1979). 


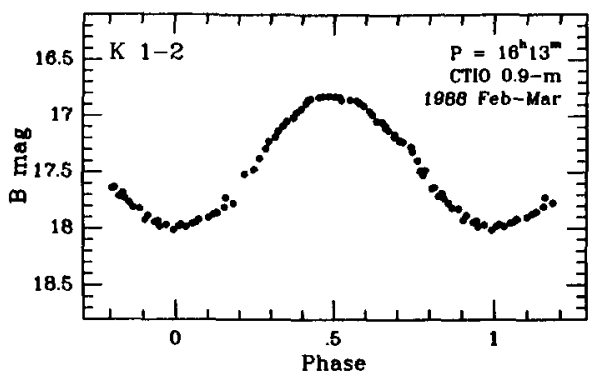

$B$ light curve for the binary nucleus of K 1-2, obtained with a CCD detector on the CTIO 36-inch telescope in February-March 1988. The orbital period is 16.2 hours, and the variations are due only to a heated hemisphere on the main-sequence secondary star; no true eclipses occur.

During its subsequent evolution, a close-binary PNN will appear first as a detached whitedwarf/red-dwarf binary (such as V471 Tau), and ultimately, when magnetic braking and/or gravitational radiation force the stars close enough together for mass transfer, as a cataclysmic variable. A discussion of the birth rate for close-binary PNNs (Bond 1988a) shows that it is, if anything, more than enough to account for the origin of all cataclysmic binaries in the solar neighborhood.

\section{Pulsating Nuclei}

Grauer and Bond (1984) discovered the first known pulsating PNN, the central star of Kohoutek 1-16. Its spectroscopic properties-strong features of He II, C IV, and O VI-are shared by the four known pulsating GW Vir (PG 1159-035) white dwarfs, and both the white dwarfs and the PNN are multiperiodic, $g$-mode pulsators. It thus seems probable that all of these objects share a common instability mechanism, possibly cyclical ionization of $\mathrm{C}$ and/or $\mathrm{O}$ in a hydrogen-deficient envelope (Starrfield et al. 1984, 1985; see critical discussion by Kawaler 1988).

The nucleus of the southern planetary nebula Longmore 4 shows strong He II and C IV absorption, without detectable Balmer absorption (Méndez et al. 1985). It was therefore an obvious candidate for photometric monitoring, and CCD photometry obtained at CTIO has now confirmed that Lo 4 is the second known pulsating PNN (Bond 1988b). A typical light curve is shown below; the dominant periodicity, as in K 1-16, is near 30 minutes, but the strong beating shows that several additional modes are present.

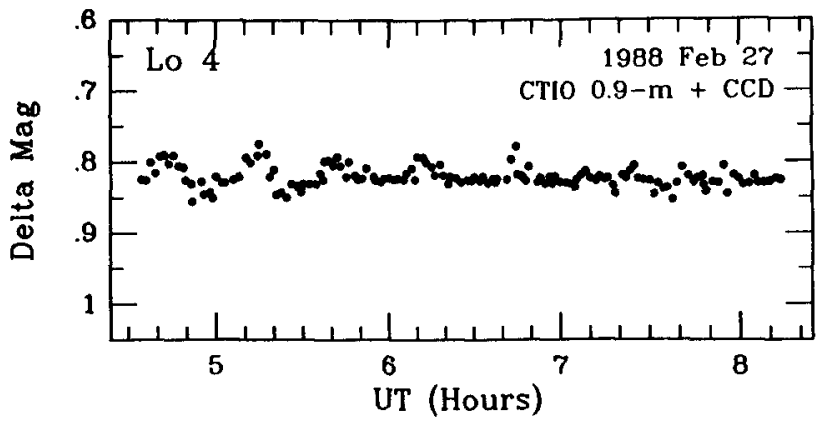

Typical CCD light curve for the pulsating central star of Longmore 4 . There is a strong periodicity near $30 \mathrm{~min}$, with beating due to several additional closely spaced frequencies. 
The pulsation periods are considerably shorter $(\sim 6-10 \mathrm{~min})$ in the four known GW Vir white dwarfs, in accordance with the view that they are somewhat more evolved, more compact stars. We presume that they were recently surrounded by planetary nebulae that have now dissipated. Continued monitoring of Lo 4 is planned, in an attempt to detect the period changes that are expected to be produced by the evolutionary contraction of this pre-white-dwarf star ( $c f$. the study of GW Vir itself by Winget et al. 1985).

Preliminary analysis of our photometry of the central star of NGC 1501, a member of the "O VI" class of PNNs, suggests that it is another pulsator; if so, its light curve is unusually chaotic, implying a very rich mode spectrum.

\section{Irregularly Variable Planetary Nuclei}

Our monitoring program has revealed several members of a new class of non-periodic, lowamplitude variables. These objects show variations of a few hundredths of a magnitude over timescales of several hours, and/or show differences in light level by such amounts from night to night. A typical light curve, for the central star of IC 4593, is shown below.

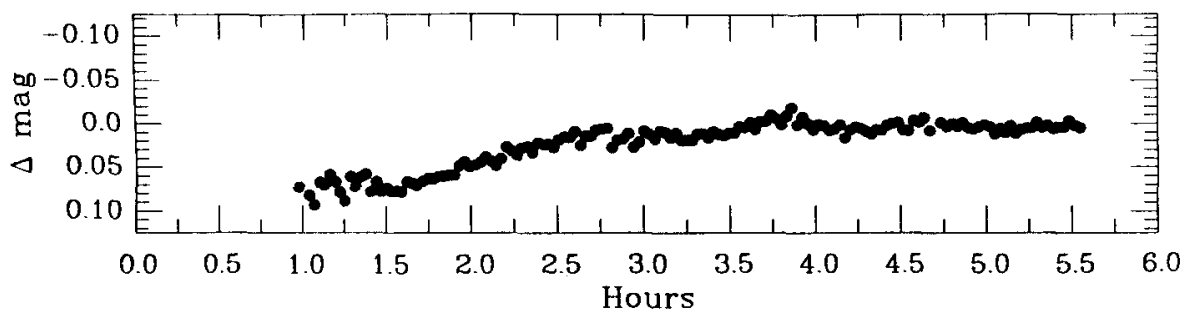

CCD light curve for the irregularly variable central star of IC 4593. During this night, the star brightened by nearly $0.1 \mathrm{mag}$ over about $3 \mathrm{hr}$, and was then nearly constant for another $1.5 \mathrm{hr}$. On other nights, the PNN varied irregularly about levels that were 0.02-0.05 mag brighter.

Other members of this class are the nuclei of $\mathrm{Hu} 2-1$, IC 3568, NGC 6543 , and possibly NGC 40. Similar photometric behavior has been reported for the central star of IC 418 by Méndez et al. (1983), and seems to be present in photoelectric photometry of IC 2149 (Bond and Grauer, unpublished).

One obvious characteristic that these variables share is the presence of a high-surfacebrightness nebula, suggesting that they are nuclei of relatively young planetaries. An even more striking characteristic is that all of them are known to have strong stellar winds, as revealed by IUE observations (Castor et al. 1981; Cerruti-Sola and Perinotto 1985).

The strong correlation of low-amplitude optical variability with presence of a stellar wind suggests either that the light variability is due to a variable mass-loss rate, or that both the variability and the mass loss arise from some common (but presently unknown) underlying physical mechanism. Further exploration of this new phenomenon will require simultaneous photometry and UV or optical spectroscopy.

\section{References}

Bond, H.E. 1988a, in Proceedings of IAU Symp. No. 131, Planetary Nebulae, in press. 1988b, Bull. A.A.S., $20,735$.

Bond, H.E., Ciardullo, R., Fleming, T.A., and Grauer, A.D. 1988, in Proceedings of IAU Symp. No. 191, Planetary Nebulae, in press. 
Bond, H.E., and Grauer, A.D. 1987, in Proceedings of IAU Colloq. No. 95, The Second Conference on Faint Blue Stars, eds. A.G.D. Philip, D.S. Hayes, and J.W. Liebert (Schenectady: L. Davis Press), p. 221.

Castor, J.I., Lutz, J.H., and Seaton, M.J. 1981, M.N.R.A.S., 194, 547.

Cerruti-Sola, M., and Perinotto, M. 1985, Ap. J., 281, 237.

Grauer, A.D., and Bond, H.E. 1984, Ap. J., 277, 211.

Kawaler, S. 1988, these proceedings.

Kohoutek, L. 1979, IAU Inf. Bull. Var. Stars, No. 1672.

Méndez, R.H., Verga, A.D., and Kriner, A. 1983, Rev. Mexicana Astr. Ap., 8, 175.

Méndez, R.H., Kudritzki, R.P., and Simon, K.P. 1985, Astr. Ap., 142, 289.

Méndez, R.H., Kudritzki, R.P., Herrero, A., Husfeld, D., and Groth, H.G. 1988, Astr. Ap., 190, 113.

Paczynski, B. 1976, in IAU Symposium No. 73, Structure and Evolution of Close-Binary Systems, eds. P. Eggleton, S. Mitton, and J. Whelan (Dordrecht: Reidel), p. 75.

Starrfield, S., Cox, A.N., Kidman, R.B., and Pesnell, W.D. 1984, Ap. J., 281, 800. 1985, Ap. J. (Letters), 293, L23.

Winget, D.E., Kepler, S.O., Robinson, E.L., Nather, R.E., and O'Donoghue, D. 1985, Ap. J., 292, 606. 